\title{
Expression of far upstream element binding protein 1 in B-cell non-Hodgkin lymphoma is correlated with tumor growth and cell-adhesion mediated drug resistance
}

\author{
YUEJIAO HUANG ${ }^{1,2^{*}}$, XIAOHONG XU ${ }^{1 *}$, LILI JI $^{3}$, YUCHAN WANG $^{1}$, SHITAO WANG $^{3}$, JIE TANG $^{2}$, \\ XIANTING HUANG ${ }^{2}$, XIAOJING YANG ${ }^{2}$, YUNHUA HE${ }^{2}$, SONG HE$^{4}$ and $\mathrm{CHUN} \mathrm{CHENG}^{2}$ \\ ${ }^{1}$ Department of Oncology, Affiliated Cancer Hospital of Nantong University, Nantong, Jiangsu 226361; \\ ${ }^{2}$ Department of Immunology, Jiangsu Province Key Laboratory for Inflammation and Molecular Drug Target; \\ ${ }^{3}$ Department of Pathology, Medical College, Nantong University, Nantong, Jiangsu 226001; ${ }^{4}$ Department of Pathology, \\ Affiliated Cancer Hospital of Nantong University, Nantong, Jiangsu 226361, P.R. China
}

Received August 8, 2015; Accepted August 9, 2016

DOI: $10.3892 / \mathrm{mmr} .2016 .5718$

\begin{abstract}
Cell adhesion-mediated drug resistance (CAM-DR) remains a major obstacle to the effectiveness of chemotherapeutic treatment of lymphoma. Far upstream element binding protein 1 (FBP1) is a multifunctional protein that is highly expressed in proliferating cells of several solid neoplasms; however, its expression and biological function in B-cell lymphoma is largely unknown. FBP1 expression in both reactive lymphoid tissues and several B-cell lymphomas, including follicular lymphoma and diffuse large B-cell lymphoma were detected by immunohistochemistry analysis. FBP1 expression in B-cell lymphoma was also associated with poor survival outcomes. Functionally, small interfering RNA-mediated silencing of FBP1 was able to inhibit the proliferation of B-cell lymphoma cells, resulting in $\mathrm{G}_{0} / \mathrm{G}_{1}$ phase cell cycle arrest. Furthermore, results of a cell adhesion assay demonstrated that adhesion to fibronectin or bone marrow stromal cells induced FBP1 expression, which in turn facilitated cell adhesion. Finally, FBP1 knockdown reversed CAM-DR. These findings support a role for FBP1 in non-Hodgkin lymphoma cell proliferation, adhesion and drug resistance, and may lead
\end{abstract}

Correspondence to: Dr Song He, Department of Pathology, Affiliated Cancer Hospital of Nantong University, 30 Tongyang Road, Nantong, Jiangsu 226361, P.R. China

E-mail: hesong515@sina.com

Dr Chun Cheng, Department of Immunology, Jiangsu Province Key Laboratory for Inflammation and Molecular Drug Target, Medical College, Nantong University, 19 Qixiu Road, Nantong, Jiangsu 226001, P.R. China

E-mail: chengchun2000@126.com

*Contributed equally

Key words: B-cell non-Hodgkin lymphoma, immunohistochemistry, proliferation, cell adhesion, drug resistance to the generation of a novel therapeutic approach targeting this molecule.

\section{Introduction}

Lymphoma is the fifth most common type of cancer; 90\% of cases of which are non-Hodgkin lymphoma (NHL) and the remaining $10 \%$ are Hodgkin lymphoma. Lymphoma is currently one of the fastest-growing cancers, with an annual increase in rate of $4-5 \%(1,2)$. NHL consists of a large group of immune system neoplasms, and represents a heterogeneous group of diseases that are characterized by the monoclonal expansion of $\mathrm{B}$ or $\mathrm{T}$ lymphocytes $(3,4)$. The classification of NHL is diverse, and includes diffuse large B-cell lymphoma (DLBCL), follicular lymphoma (FL), extranodal lymphoma of mucosa-associated lymphoid tissue (MALT) and mantle cell lymphoma $(5,6)$.

Despite marked efforts to develop novel therapeutics, and recently observed improvements in overall survival rate, which are likely due to the routine incorporation of monoclonal antibody therapy, NHL remains predominantly incurable with standard therapeutic approaches $(7,8)$. Previous studies have demonstrated that adhesion to cultured stromal cells or ligand-coated surfaces is able to protect malignant B cells from chemotherapy-induced apoptosis; this process is known as cell adhesion-mediated drug resistance (CAM-DR) $(9,10)$. Bone marrow stroma has long been known as a 'sanctuary site' for lymphoma cells during traditional chemotherapy (8). Two modes of CAM-DR have been described: i) Cell interaction, known as the stromal model, in which cells interact with a stromal cell monolayer, establishing CAM-DR by heterocellular cell interaction; ii) substrate interaction, known as the fibronectin (FN) model, in which CAM-DR is mediated by cell-substrate interaction. Although the initial type of interaction markedly differs between these models, the subsequently activated intracellular molecular mechanisms are often similar, or even identical (11).

Human far upstream element (FUSE) binding protein 1 (FBP1) was initially recognized as a factor that binds to 
the FUSE DNA sequence, which is located upstream of the c-myc proto-oncogene promoter (12). It has previously been reported that the c-myc oncogene is associated with apoptosis, growth and proliferation, and that FBP and c-myc share the same expression pattern (13). Furthermore, FBP and c-myc are expressed in proliferating cells, but not in quiescent or differentiated cells (13). FBP1 has been reported to be a potential c-myc regulator in renal cancer, but not in prostate and bladder cancer (12). In addition, several proteins, including FBP1, have been shown to be modified in Jurkat T cells during apoptosis (14). A previous study demonstrated that knockdown of FBP1 in hepatocellular carcinoma (HCC) cells resulted in increased sensitivity to apoptotic stimuli and reduced cell proliferation (15). Undoubtedly, FBP1 is a multifunctional protein; however, its function in lymphoma remains unknown.

The present study investigated the expression of FBP1 in various histological types of human B-cell NHL, and determined its prognostic role in NHL. Since stromal cell-mediated drug resistance is a common feature of chronic B-cell malignancies, including multiple myeloma and chronic lymphocytic leukemia $(16,17)$, the present study also investigated the role of FBP1 in CAM-DR in NHL. The results may provide a novel perspective for a better understanding of the mechanism underlying drug resistance in NHL.

\section{Materials and methods}

Pathological samples. The present study collected 99 B-cell lymphoma and 19 reactive lymphadenopathy (RL) biopsy samples, which were histopathologically and clinically diagnosed at the Affiliated Cancer Hospital of Nantong University (Nantong, China), between January 1, 1993 and April 1, 2005. Diagnoses were made according to the World Health Organization criteria (18). Written informed consent was obtained from all patients prior to obtaining specimens for the present study. All of the tissues were fixed with formalin and were embedded in paraffin and sectioned (3-4 $\mathrm{mm}$ ) for histopathological diagnosis and immunohistochemical study. The study was approved by the ethics committee of the Affiliated Cancer Hospital of Nantong University.

Immunohistochemistry. Immunohistochemical staining was performed with a Dako Autostainer (Dako Denmark A/S, Glostrup, Denmark) using a polymer detection system. Briefly, sections were deparaffinized in xylene and were rehydrated in a graded series of ethanol. Hydrogen peroxide $(0.3 \%)$ was used to block endogenous peroxide activity for $10 \mathrm{~min}$. Slides were then incubated with anti-FBP1 (1:900; cat. no. sc-271241; Santa Cruz Biotechnology, Inc., Dallas, TX, USA) for $4 \mathrm{~h}$ at room temperature. The tissue sections were then counterstained with hematoxylin, dehydrated and mounted.

For assessment of FBP1, >2,000 cells from five high-power fields in each specimen were selected randomly, and the staining was examined by light microscopy to determine the mean percentage. Tumor cell proportion was scored as follows: 0 , no positive tumor cells; $1,1-24 \%$ positive tumor cells; 2, 25-49\% positive tumor cells; and 3, 50-100\% positive tumor cells. Staining intensity was graded according to the following criteria: 0 , no staining; 1 , weak staining; 2 , moderate staining; and 3, strong staining (19). The product of staining intensity and the proportion of positive tumor cells was used to calculate the staining index (SI) (20). Using this method, FBP1 expression was examined in lymph node tissues by calculating the SI (scores, 0, 1, 2, 3, 4, 6 or 9). With the SI, an optimal cutoff value was identified: SI score $\geq 4$ was used to define tumors with high FBP1 expression, and SI score $\leq 3$ was used to indicate low FBP1 expression.

Cell lines, co-culture and adhesion assay. The Daudi and OCI-LY8 human malignant lymphoma cell lines were cultured in RPMI-1640 supplemented with $10 \%$ fetal bovine serum (FBS; Gibco; Thermo Fisher Scientific, Inc., Waltham, MA, USA) and $2 \mathrm{mM} \mathrm{L-glutamine} \mathrm{at} 37^{\circ} \mathrm{C}$ in an atmosphere containing $5 \% \mathrm{CO}_{2}$. The HS-5 human bone mesenchymal stem cell line was obtained from Shanghai Bioleaf Biotech Co., Ltd. (Shanghai, China), and was cultured in RPMI F12 (Sigma-Aldrich; Merck Millipore, Darmstadt, Germany). Six-well culture dishes were coated overnight at $37^{\circ} \mathrm{C}$ with a monolayer of HS- 5 cells or $5 \mu \mathrm{g} / \mathrm{cm}^{2}$ human FN (Sigma-Aldrich; Merck Millipore). Lymphoma cell lines were then allowed to adhere to the pre-established monolayer of HS-5 cells or $\mathrm{FN}$, or were maintained in suspension for $12-24 \mathrm{~h}$ at $37^{\circ} \mathrm{C}$. Subsequently, lymphoma cells were carefully removed, and the monolayer of HS-5 cells remained intact.

The Huh-7 human HCC and L02 normal liver cell lines were purchased from the Cell Library of the Chinese Academy of Science (Beijing, China). Cells were maintained in Dulbecco's modified Eagle's medium (Sigma-Aldrich; Merck Millipore) supplemented with $10 \%$ FBS, $100 \mathrm{U} / \mathrm{ml}$ penicillin, and $100 \mu \mathrm{g} / \mathrm{ml}$ streptomycin prior to use in western blot analysis to examine FBP1 expression.

The cell adhesive ability was assessed by staining lymphoma cells with calcein (Santa Cruz Biotechnology, Inc.), according to the manufacturer's protocol, for $30 \mathrm{~min}$. The cells were then incubated in 96-well plates with a FN-coated surface or pre-established monolayer of HS-5 cells in the recommended media containing $10 \%$ FBS. After $2 \mathrm{~h}$ of culture, the non-adherent cells were washed off twice with $1 \mathrm{ml}$ phosphate-buffered saline (PBS) and the number of adherent cells was measured using a fluorometer (CytoFluor; Applied Biosystems; Thermo Fisher Scientific, Inc.).

Preparation of small interfering (si)RNA and transient transfection. Daudi and OCI-LY8 cells (Jiangsu Institute of Hematology, Suzhou, China) were cultured in FBS-free RPMI 1640 medium (Sigma-Aldrich; Merck Millipore) without antibiotics. Control and FBP-siRNA (\#1, 5'-GGTGCTGAC AAACCTCTT-3'; \#2, 5'-CCCATATAAAGTTCAACA-3'; and \#3, 5'-GCTGCTTATTACGCTCAC-3') were purchased from Shanghai GenePharma Co., Ltd. (Shanghai, China) and transfection of cells with duplex synthetic siRNA was performed with Lipofectamine 2000 (Invitrogen; Thermo Fisher Scientific, Inc.) according to the manufacturer's protocol. After $48 \mathrm{~h}$, cells were resuspended in normal medium at $10^{6} / \mathrm{ml}$ and processed for further experiments.

Immunoblot analysis. The OCI-LY8 and Daudi cells, and lymph node tissues were homogenized in lysis buffer [1\% NP-40, $50 \mathrm{mmol} / 1$ Tris (pH 7.5), $5 \mathrm{mmol} / 1$ EDTA, $1 \%$ sodium dodecyl sulfate (SDS), $1 \%$ sodium deoxycholate, 

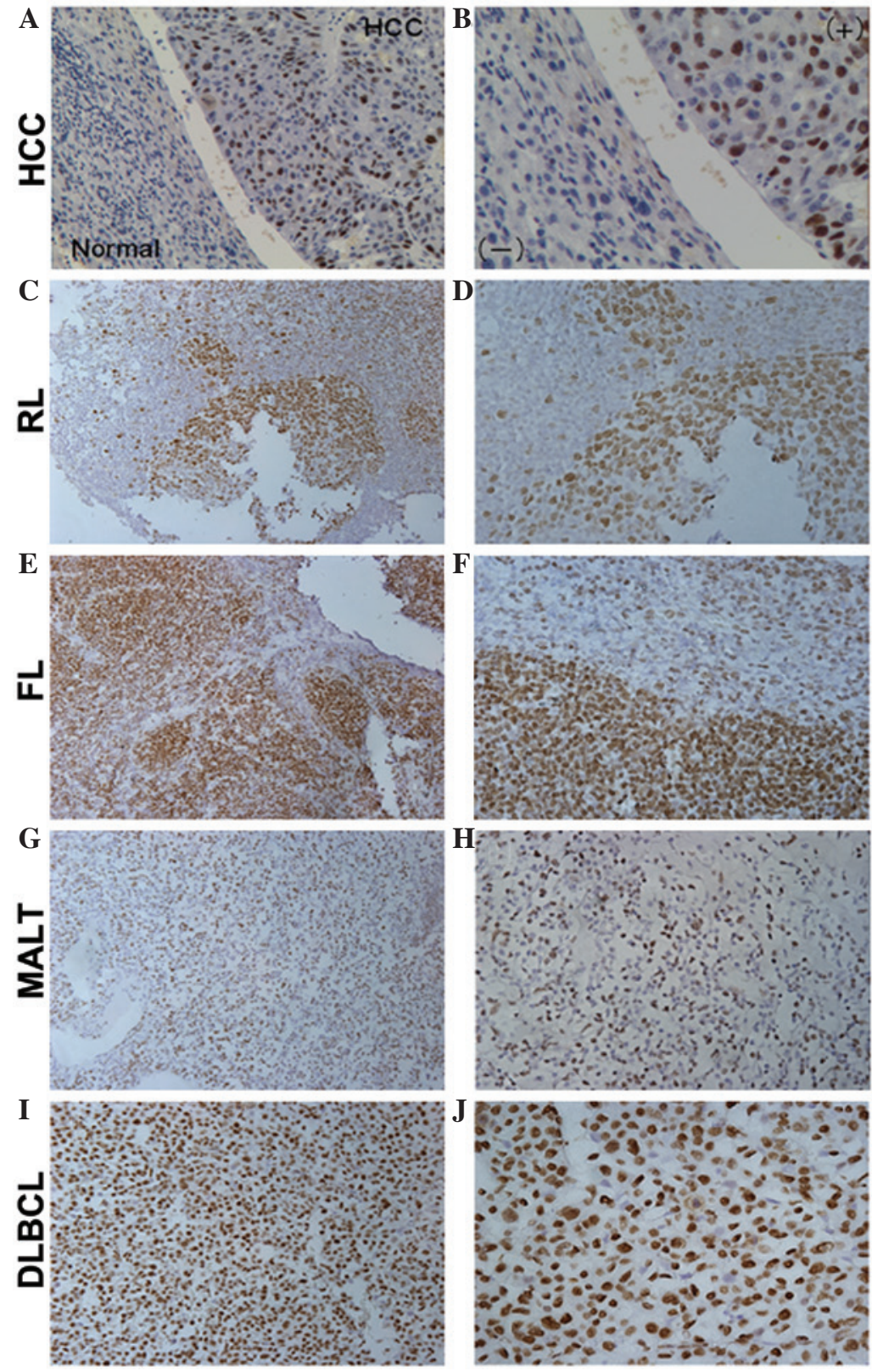

Figure 1. Immunohistochemical staining (IHC) results for far upstream element binding protein 1 (FBP1) expression in reactive lymphadenopathy (RL) and human B-cell non-Hodgkin lymphoma tissues. IHC was performed to detect FBP1 expression in (A and B) hepatocellular carcinoma (HCC) and adjacent normal tissues, as the positive and negative controls (A, x20; B, x40); (C and D) RL (C, x20; D, x40); (E and F) follicular lymphoma (FL; E, x20; F, x40); $(\mathrm{G}$ and $\mathrm{H}$ ) extranodal lymphoma of mucosa-associated lymphoid tissue (MALT; G, x20; H, x40) and (I and J) diffuse large B-cell lymphoma (DLBCL; I, x20; $\mathrm{J}, \mathrm{x} 40$ ). The FBP1 expression pattern varied between the different lymphoma types (DLBCL vs. FL vs. MALT=63.88\% vs. $35.29 \%$ vs. $40.74 \%$ ).

$1 \%$ Triton X-100, $1 \mathrm{mmol} / \mathrm{l}$ phenylmethylsulfonyl fluoride, $10 \mathrm{~g} / \mathrm{ml}$ aprotinin and $1 \mathrm{~g} / \mathrm{ml}$ leupeptin] and were cleared by centrifugation at $9,388 \times g$ for $20 \mathrm{~min}$ in a microcentrifuge at $4^{\circ} \mathrm{C}(21)$. A nuclei acid analyzerwas used to determine the protein concentration and equal amounts of total protein $(100 \mu \mathrm{g})$ were separated by $10 \%$ SDS-polyacrylamide gel electrophoresis and were electrophoretically transferred to polyvinylidene fluoride membranes (EMD Millipore, Bedford, MA, USA). The membranes were then blocked with $5 \%$ nonfat milk and were incubated with anti-FBP1 (1:1,000); anti-cyclin-dependent kinase 2 (CDK2; 1:500; cat. no. sc-163); anti-cyclin A (1:500; cat. no. sc-751; ); anti-cyclin D1 (1:500; cat. no. sc-753); anti-proliferating cell nuclear antigen (PCNA; 1:1,000; cat. no. sc-790); anti-c-myc (1:1,000; cat. no. sc-764); anti-glyceraldehyde 3-phosphate dehydrogenase (GAPDH; 1:1,000; cat. no. sc-32233), all obtained from Santa Cruz Biotechnology, Inc.; and anti-cleaved caspase $(1: 2,000$; cat. no. C8487; Sigma-Aldrich; Merck Millipore) antibodies overnight at $4^{\circ} \mathrm{C}$. Following three washes with Tris-buffered saline- $0.1 \%$ Tween 20 , the blots were incubated with horseradish peroxidase-conjugated secondary antibodies (1:10,000; cat. no. PV-6000-D; OriGene Technologies, Inc., Beijing, China) for $2 \mathrm{~h}$ at room temperature. The signals were visualized using an enhanced chemiluminescence detection system. Protein levels were analyzed using Image J (version 1.49; imagej.nih.gov/ij/) and normalized with GAPDH levels. The results were derived from at least three independent experiments. 
Table I. FBP1 expression and clinicopathological parameters in 99 non-Hodgkin lymphoma specimens.

\begin{tabular}{|c|c|c|c|c|}
\hline \multirow[b]{2}{*}{ Parameter } & \multirow[b]{2}{*}{ Total } & \multicolumn{2}{|c|}{ FBP1 expression } & \multirow[b]{2}{*}{ P-value } \\
\hline & & Low & High & \\
\hline Age (years) & & & & $<0.001^{\mathrm{a}}$ \\
\hline$\leq 60$ & 59 & 36 & 23 & \\
\hline$>60$ & 40 & 11 & 29 & \\
\hline Gender & & & & 0.743 \\
\hline Male & 56 & 30 & 26 & \\
\hline Female & 43 & 17 & 26 & \\
\hline B symptoms & & & & 0.967 \\
\hline Absent & 20 & 11 & 9 & \\
\hline Present & 79 & 36 & 43 & \\
\hline Extranodal sites & & & & $0.025^{\mathrm{b}}$ \\
\hline$<2$ & 64 & 35 & 29 & \\
\hline$\geq 2$ & 35 & 12 & 23 & \\
\hline Lactate dehydrogenase $\mathrm{e}^{\mathrm{b}}$ & & & & 0.557 \\
\hline Normal & 30 & 17 & 13 & \\
\hline Elevated & 33 & 11 & 22 & \\
\hline Treatment & & & & 0.224 \\
\hline $\mathrm{CHOP}$ & 55 & 24 & 31 & \\
\hline Other & 44 & 22 & 22 & \\
\hline Ki-67 expression & & & & $<0.001^{\mathrm{b}}$ \\
\hline$<70 \%$ & 42 & 33 & 9 & \\
\hline$\geq 70 \%$ & 57 & 11 & 46 & \\
\hline Histological type & & & & 0.482 \\
\hline Indolent & 63 & 39 & 24 & \\
\hline Invasive & 36 & 13 & 23 & \\
\hline
\end{tabular}

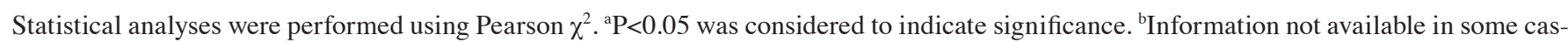
es. CHOP, cyclophosphamide, doxorubicin hydrochloride, vincristine, prednisolone treatment; FBP1, far upstream element binding protein 1.

Cell cycle analysis and viability assay. OCI-LY8 and Daudi cells were fixed in $75 \%$ ethanol at $-20^{\circ} \mathrm{C}$ overnight. Subsequently, the cells were incubated with $1 \mathrm{mg} / \mathrm{ml}$ RNase A for $30 \mathrm{~min}$, and were stained with propidium iodide (PI; $50 \mu \mathrm{g} / \mathrm{ml}$; BD Biosciences, San Jose, CA, USA) in PBS containing $0.5 \%$ Tween-20. Cells were analyzed using a BD FACScan flow cytometer (BD Biosciences).

Cell proliferation and viability were assessed using the standard Cell Counting kit-8 (CCK-8; Dojindo Molecular Technologies, Inc., Rockville, MD, USA), according to the manufacturer's protocol. The viability of lymphoma cells following treatment with $1 \mu \mathrm{M}$ doxorubicin (Sigma-Aldrich; Merck Millipore) for 48 or $72 \mathrm{~h}$ was determined by CCK- 8 assay.

Statistical analysis. SPSS 15.0 software (SPSS, Inc., Chicago, IL, USA) was used to perform statistical analysis. Differences between two groups were compared using $\chi^{2}$ test (Table I). Multivariate analysis was performed using Cox's proportional hazards model (Table II). The risk ratio and its $95 \%$ confidence interval were recorded for each marker. Other statistical analyses were performed using the Student's t-test. $\mathrm{P}<0.05$ was considered to indicate a statistically significant difference. Each experiment consisted of at least three replicates per condition.

\section{Results}

FBPI is expressed in RL and human B-cell NHL tissues. FBP1 is highly expressed in several solid neoplasms, including basal-like breast cancer, renal cancer, HCC, colon cancer and non-small cell lung cancer $(12,22-25)$; however, whether this is true for hematological malignancies remains to be elucidated. Therefore, immunohistochemical analysis was performed to investigate the in vivo expression of FBP1 in clinical specimens of RL and lymphoma tissues, including FL, MALT and DLBCL (Fig. 1). HCC tissue was used as a positive control, whereas adjacent normal tissue was used as a negative control (15) (Fig. 1A and B). In RL tissues, FBP1 was predominantly expressed in proliferating germinal centers (Fig. 1C and D). Furthermore, in lymphoma tissues other than MALT, overexpression of FBP1 was detected. FBP1 
Table II. Multivariate analysis with Cox regression model of 99 non-Hodgkin lymphoma specimens.

\begin{tabular}{|c|c|c|c|}
\hline Parameter & Relative ratio & $95 \%$ confidence interval & P-value \\
\hline Age (years) & & & $<0.01^{\mathrm{a}}$ \\
\hline$>60$ & 1.114 & $0.434-0.717$ & \\
\hline Gender & & & 0.615 \\
\hline Female & 0.842 & $0.504-0.615$ & \\
\hline B symptoms & & & $0.019^{\mathrm{a}}$ \\
\hline Present & 0.723 & $0.048-0.419$ & \\
\hline Extranodal sites & & & $<0.01^{\mathrm{a}}$ \\
\hline$\geq 2$ & 0.967 & $0.157-0.514$ & \\
\hline Lactate dehydrogenase $^{\mathrm{b}}$ & & & $0.045^{\mathrm{a}}$ \\
\hline Elevated & 0.924 & $0.011-0.404$ & \\
\hline Treatment & & & 0.598 \\
\hline $\mathrm{CHOP}$ & 0.979 & $0.135-0.247$ & \\
\hline Ki-67 expression & & & $<0.01^{\mathrm{a}}$ \\
\hline$\geq 70 \%$ & 1.156 & $0.420-0.717$ & \\
\hline Histological type & & & $0.006^{\mathrm{a}}$ \\
\hline Invasive & 1.949 & $0.096-0.447$ & \\
\hline FBP1 expression & & & $<0.01^{\mathrm{a}}$ \\
\hline High & 1.138 & $0.521-0.766$ & \\
\hline
\end{tabular}

Statistical analyses were performed using Cox regression test. ${ }^{\mathrm{a}} \mathrm{P}<0.05$ was considered to indicate significance. ${ }^{\mathrm{b}}$ Information not available in some cases. CHOP, cyclophosphamide, doxorubicin hydrochloride, vincristine, prednisolone treatment; FBP1, far upstream element binding protein 1 .

immunoreactivity was primarily localized in the follicular mantle zones of FL (Fig. 1E and F), whereas in DLBCL tissues FBP1 was diffusely expressed (Fig. 1I and J). Compared with FL and DLBCL tissues, the expression of FBP1 in MALT tissues was much weaker (Fig. $1 \mathrm{G}$ and H). Furthermore, the positive rate of FBP1 expression was evaluated among the subtypes of lymphoma. FBP1 was highly expressed in several lymphoma tissues, with $63.88 \%$ positivity in DLBCL (23/36), 35.29\% positivity in FL (6/17), and 40.74\% positivity in MALT (11/27). Generally speaking, the FBP1 expression pattern varied among the various lymphoma types; however, there were no quantifiable differences between expression of FBP1 between tumor types, or between malignant and non-malignant specimens $(\mathrm{P}=0.482)$.

FBP1 expression is associated with high-risk clinical parameters in NHL. Various clinicopathological parameters were compared between patients with high or low FBP1 expression (Table I). A significant positive correlation was detected between FBP1 expression and Ki-67 $(\mathrm{P}<0.001)$, which is a proliferative marker. There were also significant correlations between high levels of FBP1 expression and two other adverse prognostic factors: Advanced age $(\mathrm{P}<0.001)$ and multiple extranodal sites $(\mathrm{P}<0.05)$. However, high levels of FBP1 expression were not significantly correlated with gender, serum lactate dehydrogenase (LDH) levels, histological type, chemotherapy or clinical symptoms. Survival analysis was performed on 99 patients who had follow-up data until mortality. After all variables were compared separately with survival status,
FBP1 $(\mathrm{P}<0.01)$ Ki-67 $(\mathrm{P}<0.01)$, age $(\mathrm{P}<0.01)$, B symptoms $(\mathrm{P}=0.019)$, extranodal sites $(\mathrm{P}<0.01), \mathrm{LDH}(\mathrm{P}=0.045)$ and invasive histological type $(\mathrm{P}=0.006)$ significantly influenced survival (Table II).

FBPI expression promotes proliferation of NHL cell lines. According to a previous study (23), FBP1 can be detected in HCC cell lines, including Huh-7; however, it is undetectable in the L02 normal liver cell line. Therefore, the present study assessed the protein expression levels of FBP1 in three cell lines: OCI-LY8, and the positive and negative control cells, Huh-7 and L02 (Fig. 2A). To further investigate the potential effects of FBP1 on NHL cell proliferation, OCI-LY8 cells were transiently transfected with FBP1-siRNA for $48 \mathrm{~h}$, and the efficacy of FBP1 siRNA-mediated downregulation was confirmed by western blot analysis (Fig. 2B). Of the three siRNAs used FBP1-siRNA\#1 exhibited the highest efficacy (Fig. 2B). Therefore, FBP1-siRNA\#1 was chosen for subsequent assays. OCI-LY8 cells were transfected with FBP1-siRNA\#1, and the expression levels of FBP1, c-myc, PCNA, CDK2, cyclin A and cyclin D1 were measured by western blotting (Fig. 2C). Previous studies have suggested that FBP1 is associated with carcinogenesis via c-myc dependent or independent pathways $(15,26)$. The present study demonstrated that the expression levels of c-myc were higher when FBP1 expression was not suppressed. Knockdown of FBP1 resulted in a marked decrease in the expression of PCNA, which is a marker of cell proliferation. Furthermore, knockdown of FBP1 was correlated with the downregulation of several key cell cycle regulators, including 
A

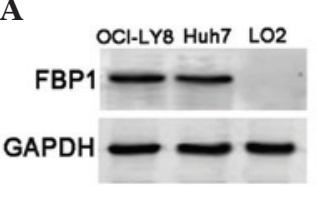

B
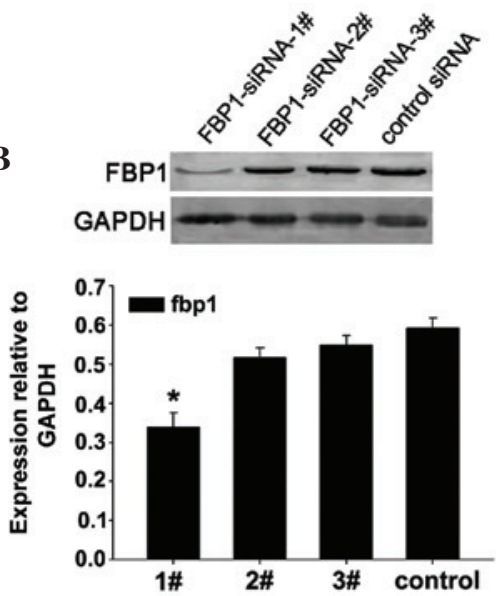

C

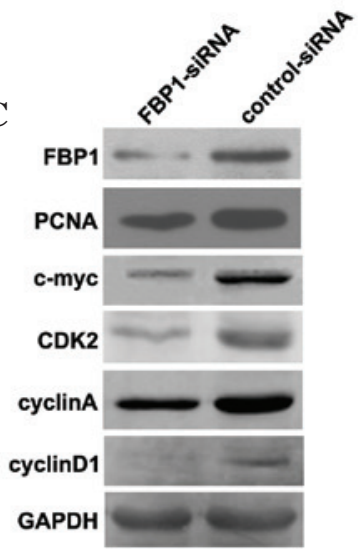

D


Figure 2. Far upstream element binding protein 1 (FBP1) expression promotes proliferation of OCI-LY8 cells. (A) Expression of FBP1 in various cell lines. The hepatocellular carcinoma cell line Huh-7 was used as a positive control, and the normal liver cell line L02 was used as a negative control. (B) OCI-LY8 cells were transfected with either FBP1-small interfering (si)RNA or a scrambled sequence (control siRNA). The efficacy of FBP1-siRNA-mediated downregulation was conformed by western blot analysis, with glyceraldehyde 3-phosphate dehydrogenase (GAPDH) as a loading control. "P<0.05 vs. control siRNA. (C) OCI-LY8 cells transfected with FBP1-siRNA or control siRNA were lysed and analyzed by western blotting using antibodies against FBP1, c-myc, proliferating cell nuclear antigen (PCNA), cell-dependent kinase 2 (CDK2), cyclin A, cyclin D1 and GAPDH. (D) Cell growth was examined by Cell Counting kit-8 assay at the indicated times. Each experiment was conducted at least three times. (E) Cells transfected with FBP1-siRNA or control siRNA were stained with propidium iodide for DNA content analysis by flow cytometry. OD, optical density.

CDK2, cyclin A and cyclin D1. Subsequently, a CCK8 assay was performed to determine cell viability of cells transfected with FBP1-siRNA (Fig. 2D). Knockdown of FBP1 resulted in a marked inhibition of cell growth rate. To explore the mechanism underlying FBP1-siRNA-induced decreased cell growth, cell cycle distribution was determined following transfection with FBP1-siRNA or control siRNA by flow cytometry. The percentage of cells in $\mathrm{S}$ phase was markedly decreased in the FBP1-siRNA group compared with in the control siRNA group (Fig. 2E), thus suggesting that FBP1 may promote $G_{0} / G_{1}-S$ transition and accelerate cell growth. Similar results were detected in the experiments that used the Daudi cell line (data not shown).

Adhesion to FN or bone marrow stromal cells induces FBPI protein expression, which in turn facilitates cell adhesion. It has previously been demonstrated that adhesion of hematological malignant cells to the extracellular matrix component FN reverses cell cycle arrest (27). The present study conducted an adhesion assay where lymphoma cells were adhered to 
A

Daudi

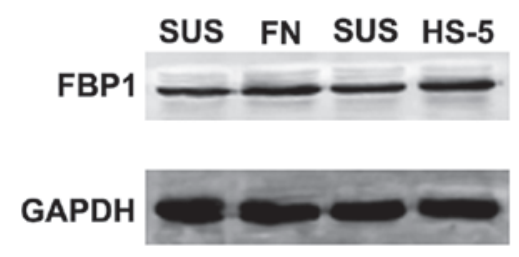

OCI-LY8

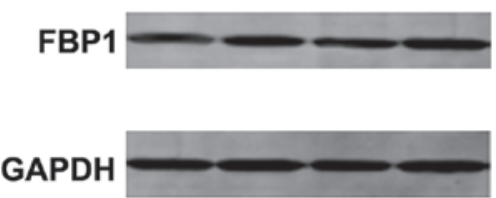

B
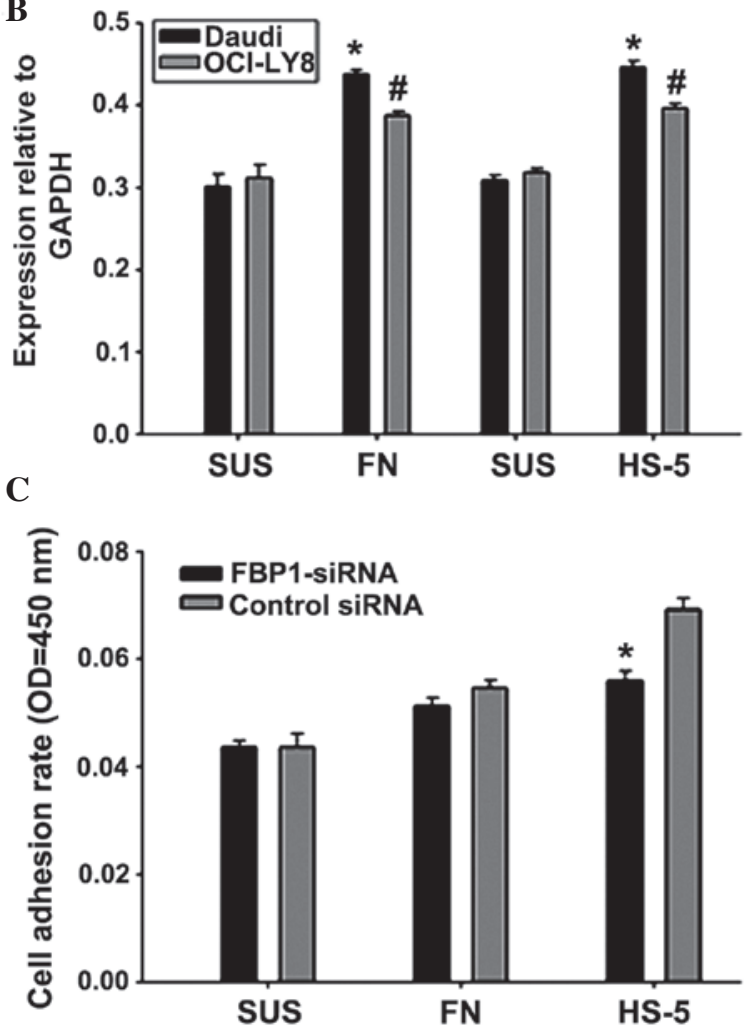

Figure 3. Adhesion to fibronectin (FN) or bone marrow stroma induces far upstream element binding protein 1 (FBP1) protein expression, which in turn facilitates cell adhesion. (A) FBP1 expression in Daudi and OCI-LY8 cells adherent to stromal cells, adeherent to FN, or in suspension (SUS). (B) Ratio of FBP1 protein to glyceraldehyde 3-phosphate dehydrogenase (GAPDH), as determined by densitometry. Each experiment was repeated at least three times. Data are presented as the mean \pm standard error of the mean. " $\mathrm{P}<0.05$, vs. Daudi cells in suspension; ${ }^{*} \mathrm{P}<0.05$ vs. OCI-LY cells in suspension. (C) Cells were plated on a FN-coated surface or a pre-established monolayer of HS-5 cells and were stained with calcein. After $2 \mathrm{~h}$ the non-adherent cells were washed twice, and the number of adherent cells was measured. Cell adhesion rate was significantly higher in the control small interfering (si)RNA group compared with in the FBP1-siRNA group. " $\mathrm{P}<0.05$ vs. control siRNA. OD, optical density.

stromal cells or FN in an attempt to elucidate the correlation between cell adhesion and the expression of FBP1. A western blot analysis was conducted to evaluate the expression of FBP1 in Daudi and OCI-LY8 cells following $24 \mathrm{~h}$ of adhesion to stromal cells or $\mathrm{FN}$, or in suspension. FBP1 protein expression was markedly increased when lymphoma cells adhered to FN or stromal cells compared with cells in suspension (Fig. 3A and B). Subsequently, the role of FBP1 in promoting adhesion of lymphoma cells to FN or HS-5 cells was investigated using FBP1-siRNA. When cells were cultured in the presence of FN or HS-5, the adhesion rates of Daudi cells were more significantly increased compared with OCI-LY8 cells. Therefore, Daudi cells were transiently transfected with FBP1-siRNA or control siRNA for $48 \mathrm{~h}$ and were stained with calcein for $30 \mathrm{~min}$. Subsequently, they were plated on a FN-coated surface or pre-established monolayers of HS-5 and were cultured for a further $2 \mathrm{~h}$. Cell adhesion assay revealed that the cell adhesion rate was significantly reduced following knockdown of FBP1 in the HS-5 cell adhesion group (Fig. 3C). Taken together, the expression of FBP1 may promote the adhesion of Daudi cells to FN or HS-5 cell, which may lead to CAM-DR.

FBP1 knockdown reverses adhesion-mediated drug resistance. CAM-DR is considered a major mechanism by which tumor cells escape the cytotoxic effects of therapeutic agents $(28,29)$. The present study demonstrated that FBP1 expression promotes adhesion of lymphoma cells. However, the specific association of FBP1 with drug resistance has yet to be elucidated. Therefore, FBP1-siRNA was used to knock down FBP1 expression in Daudi lymphoma cells cultured in three different conditions: Adhesion to FN, adhesion to bone marrow stromal cells, or in suspension. Subsequently, in order to evaluate the effects of FBP1 downregulation on drug resistance, a CCK8 assay was conducted following treatment with doxorubicin for 48 or $72 \mathrm{~h}$ (Fig. 4A and B). In a preliminary experiment, the appropriate drug concentration for treatment of lymphoma cells was determined to be $1 \mu \mathrm{M}$; this was the concentration at which cells were shown to be sensitive to drug-induced apoptosis. The results of the present study demonstrated that cell adhesion to HS-5 cells significantly protected Daudi lymphoma cells from the cytotoxicity of doxorubicin, as compared with cells in suspension. Conversely, this effect was partially abrogated following knockdown of FBP1. Adhesion to FN only resulted in weak drug resistance, and there was no marked downregulation of drug resistance in FN-adhered cells following FBP1-siRNA transfection. These results support a role for FBP1 in conferring drug resistance through cell-adhesion mechanisms.

It has previously been reported that FBP1 can be cleaved in the process of chemotherapy-induced apoptosis (13). Therefore, cleavage of FBP1, along with caspase- 3 was evaluated by western blot analysis (Fig. 4C). Exposure of Daudi 

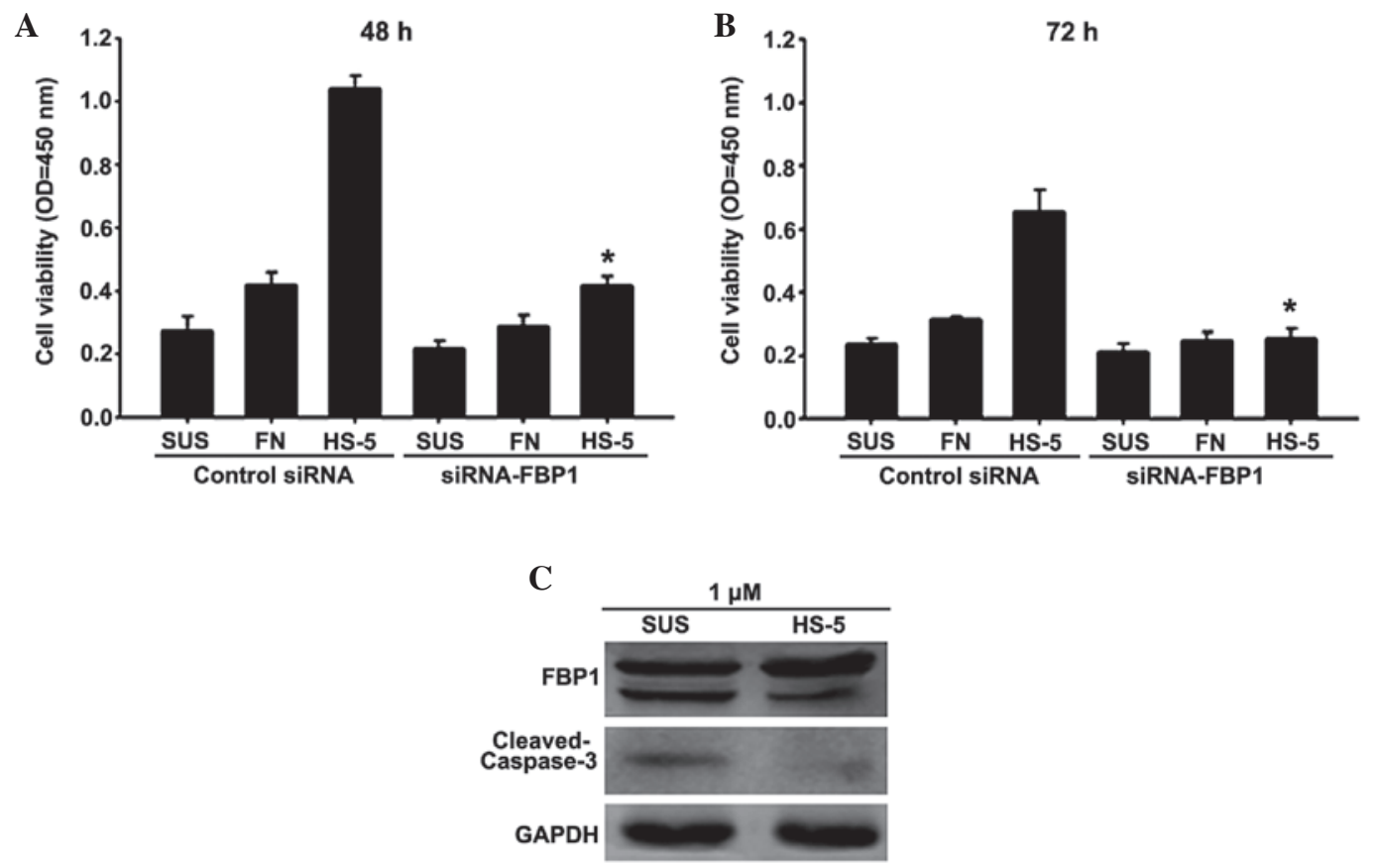

Figure 4. Far upstream element binding protein 1 (FBP1) knockdown reverses adhesion-mediated drug resistance. (A and B) Daudi cells were transfected with small interfering (si)RNA targeting either FBP1 or a scrambled sequence (control siRNA). The cells were then cultured in three conditions: Adhesion to fibronectin (FN), adhesion to bone marrow stromal cells (HS-5), or in suspension (SUS), alongside doxorubicin treatment. Cell Counting kit-8 assay was performed to evaluate the effects of FBP1 knockdown on drug resistance following treatment with $1 \mu \mathrm{M}$ doxorubicin for 48 or $72 \mathrm{~h}$. Data are representative of three independent experiments. (C) Cleavage of FBP1 and the apoptosis marker caspase-3 in Daudi cells adhered to HS-5 cells or in suspension was evaluated by western blotting. Daudi cells were treated with $1 \mu \mathrm{M}$ doxorubicin. Each experiment was performed at least three times. "P $<0.05$ siRNA-FBP1 vs. control siRNA. GAPDH, glyceraldehyde 3-phosphate dehydrogenase; OD, optical density.

lymphoma cells to $1 \mu \mathrm{M}$ doxorubicin resulted in cleavage of FBP1 and caspase-3. Cell adhesion to HS-5 cells led to decreased cleavage of FBP1 and caspase-3, thus suggesting that stromal cell adhesion inhibited drug-induced apoptosis. Furthermore, compared with cells in suspension, adhesion to HS-5 cells increased FBP1 expression, which was consistent with previous results. These findings suggest that cell-cell interactions can substitute for cell-substrate interactions in conferring apoptotic resistance in NHL cells. FBP1 has an important role in stromal/lymphoma cell interactions and may be considered a novel therapeutic target for residual resistant lymphoma following chemotherapy.

\section{Discussion}

NHL is a disease that demonstrates a high proliferative rate and eventually becomes resistant to chemotherapy (30). Lymphoma cells are present in the bone marrow or secondary lymphoid organs, thus suggesting that the microenvironment may provide necessary components for growth and survival of tumor cells (31). FBP1, which acts as an activator of transcription for the proto-oncogene c-myc, contributes to decreased cell sensitivity to apoptotic stimuli and increased cell proliferation (23). FBP1 is a potential target of malignant cell transformation. It has been found to be upregulated in several types of cancer, including basal-like breast cancer, renal cancer, HCC, colon cancer and non-small cell lung cancer $(15,22-26)$. These findings have implicated FBP1 expression in the progression of solid cancers; however, whether FBP1 is involved in hematological malignancies remains largely unknown. The present study detected FBP1 expression in RL tissues and several types of B-cell lymphoma tissue, including FL and DLBCL, by immunohistochemical analysis. In addition, FBP1 expression was shown to be associated with high-risk clinical parameters, and was identified as an independent prognostic factor for NHL in multivariate analysis. Therefore, evaluation of FBP1 expression may be considered an important factor in identifying poor prognosis in patients with NHL.

To explore the mechanisms underlying the effects of FBP1 on the promotion of NHL cell proliferation, knockdown of FBP1 via FBP1-siRNA transfection was performed in OCI-LY8 and Daudi cells. Downregulation of FBP1 expression significantly decreased the protein expression levels of cyclin A, cyclin D1 and CDK2, and suppressed the cell cycle progression of OCI-LY8 cells. It is well-known that $\mathrm{G}_{1} / \mathrm{S}$ phase transition is a major checkpoint for cell cycle progression, and two complexes, cyclin D1-CDK4 and cyclin E-CDK2, function as critical positive regulators during this transition (32). Accordingly, the results of the present study indicated that the expression levels of cyclin D1 and CDK2 were decreased in FBP1-siRNA-transfected OCI-LY8 cells. These results suggested that FBP1 may be considered a promising novel target for the treatment of NHL therapy.

A better understanding regarding the biology of B-cell malignancies is required for the development of potential therapeutic agents that target specific intracellular pathways and the interaction between malignant $\mathrm{B}$ cells and their microenvironment (33). The 'tumor microenvironment' is a critical determinant for tumor initiation, progression, response to therapy, and drug resistance. Previous studies, including results from our own 
laboratory, have reported that cell adhesion of hematopoietic tumor cell lines to stromal cells confers a multidrug-resistant phenotype, and that disruption of cell adhesion-mediated signaling may increase the efficacy of currently used cytotoxic agents (34-36). The present study investigated the effects of adhesion between stromal cells and B-cell lymphoma cells on the survival of NHL cell lines. The results indicated that FBP1 may have an important role in the adhesion and survival of NHL cells. Downregulation of FBP1 expression attenuated the observed CAM-DR. Therefore, a better understanding regarding the molecular mechanisms underlying the effects of FBP1 on stromal/lymphoma cell interactions may lead to the generation of novel therapeutic approaches for the treatment of residual resistant lymphoma following chemotherapy. However, in the process of CAM-DR, it remains unclear as to which signal pathway or targets are affected by FBP1 expression; therefore, this mechanism may warrant further investigation.

In conclusion, the present study demonstrated that FBP1 has a critical role in NHL cell proliferation, adhesion and drug resistance. These results may lead to the generation of a novel therapeutic approach that targets FBP1.

\section{Acknowledgements}

The present study was supported by grants from the National Natural Science Foundation of China (grant nos. 81172879, 81201858 and 81372537); the Natural Scientific Foundation of Jiangsu Province Grant (grant no. BK2012231); the National Funds for Distinguished Young Scientists of Nantong City (grant no. WQ2016057); and a Project Funded by the Priority Academic Program Development of Jiangsu Higher Education Institutions (PAPD).

\section{References}

1. Lwin T, Hazlehurst LA, Li Z, Dessureault S, Sotomayor E, Moscinski LC, Dalton WS and Tao J: Bone marrow stromal cells prevent apoptosis of lymphoma cells by upregulation of anti-apoptotic proteins associated with activation of NF-kappaB (RelB/p52) in non-Hodgkin's lymphoma cells. Leukemia 21: 1521-1531, 2007.

2. Ding BB, Yu JJ, Yu RY, Mendez LM, Shaknovich R, Zhang Y, Cattoretti $\mathrm{G}$ and Ye BH: Constitutively activated STAT3 promotes cell proliferation and survival in the activated B-cell subtype of diffuse large B-cell lymphomas. Blood 111: 1515-1523, 2008.

3. Wang K, Jiang Y, Zheng W, Liu Z, Li H, Lou J, Gu M and Wang $X$ : Silencing of human phosphatidylethanolamine-binding protein 4 enhances rituximab-induced death and chemosensitization in B-cell lymphoma. PLoS One 8: e56829, 2013.

4. Wang Y, Fei M, Cheng C, Zhang D, Lu J, He S, Zhao Y, Wang $Y$ and Shen A: Jun activation domain-binding protein 1 negatively regulate p27 kip1 in non-Hodgkin's lymphomas. Cancer Biol Ther 7: 460-467, 2008.

5. Hang Q, Fei M, Hou S, Ni Q, Lu C, Zhang G, Gong P, Guan C, Huang $X$ and He S: Expression of Spyl protein in human non-Hodgkin's lymphomas is correlated with phosphorylation of p27 Kip1 on Thr187 and cell proliferation. Med Oncol 29: 3504-3514, 2012.

6. Zhao Y, Fei M, Wang Y, Lu M, Cheng C and Shen A: Expression of Foxo3a in non-Hodgkin's lymphomas is correlated with cell cycle inhibitor p27. Eur J Haematol 81: 83-93, 2008.

7. Lwin T, Lin J, Choi YS, Zhang X, Moscinski LC, Wright KL, Sotomayor EM, Dalton WS and Tao J: Follicular dendritic cell-dependent drug resistance of non-Hodgkin lymphoma involves cell adhesion-mediated Bim down-regulation through induction of microRNA-181a. Blood 116: 5228-5236, 2010.
8. Lwin T, Crespo LA, Wu A, Dessureault S, Shu HB, Moscinski LC, Sotomayor E, Dalton WS and Tao J: Lymphoma cell adhesion-induced expression of $\mathrm{B}$ cell-activating factor of the TNF family in bone marrow stromal cells protects non-Hodgkin's B lymphoma cells from apoptosis. Leukemia 23: 170-177, 2009.

9. Mraz M, Zent CS, Church AK, Jelinek DF, Wu X, Pospisilova S, Ansell SM, Novak AJ, Kay NE, Witzig TE and Nowakowski GS: Bone marrow stromal cells protect lymphoma B-cells from rituximab-induced apoptosis and targeting integrin alpha-4- $\beta-1$ (VLA-4) with natalizumab can overcome this resistance. Br J Haematol 155: 53-64, 2011.

10. Hazlehurst LA, Argilagos RF, Emmons M, Boulware D, Beam CA, Sullivan DM and Dalton WS: Cell adhesion to fibronectin (CAM-DR) influences acquired mitoxantrone resistance in U937 cells. Cancer Res 66: 2338-2345, 2006.

11. Westhoff MA, Zhou S, Bachem MG, Debatin KM and Fulda S: Identification of a novel switch in the dominant forms of cell adhesion-mediated drug resistance in glioblastoma cells. Oncogene 27: 5169-5181, 2008.

12. Weber A, Kristiansen I, Johannsen M, Oelrich B, Scholmann K, Gunia S, May M, Meyer HA, Behnke S, Moch H and Kristiansen G: The FUSE binding proteins FBP1 and FBP3 are potential c-myc regulators in renal, but not in prostate and bladder cancer. BMC Cancer 8: 369, 2008.

13. Jang M, Park BC, Kang S, Chi SW, Cho S, Chung SJ, Lee SC, Bae KH and Park SG: Far upstream element-binding protein-1, a novel caspase substrate, acts as a cross-talker between apoptosis and the c-myc oncogene. Oncogene 28: 1529-1536, 2009.

14. Thiede B, Dimmler C, Siejak F and Rudel T: Predominant identification of RNA-binding proteins in Fas-induced apoptosis by proteome analysis. J Biol Chem 276: 26044-26050, 2001.

15. Rabenhorst U, Beinoraviciute-Kellner R, Brezniceanu ML, Joos S, Devens F, Lichter P, Rieker RJ, Trojan J, Chung HJ, Levens DL and Zörnig M: Overexpression of the far upstream element binding protein 1 in hepatocellular carcinoma is required for tumor growth. Hepatology 50: 1121-1129, 2009.

16. Hazlehurst LA, Damiano JS, Buyuksal I, Pledger WJ and Dalton WS: Adhesion to fibronectin via betal integrins regulates p27kip1 levels and contributes to cell adhesion mediated drug resistance (CAM-DR). Oncogene 19: 4319-4327, 2000.

17. Shain KH, Yarde DN, Meads MB, Huang M, Jove R, Hazlehurst LA and Dalton WS: Beta1 integrin adhesion enhances IL-6-mediated STAT3 signaling in myeloma cells: Implications for microenvironment influence on tumor survival and proliferation. Cancer Res 69: 1009-1015, 2009.

18. Naz E, Mirza T, Aziz S, Danish F, Siddiqui ST and Ali A: Frequency and clinicopathologic correlation of different types of non Hodgkin's lymphoma according to WHO classification. J Pak Med Assoc 61: 260-263, 2011.

19. Filtenborg-Barnkob BE and Bzorek M: Expression of anaplastic lymphoma kinase in Merkel cell carcinomas. Hum Pathol 44: 1656-1664, 2013.

20. Li M, Yang X, Shi H, Ren H, Chen X, Zhang S, Zhu J and Zhang J: Downregulated expression of the cyclase-associated protein 1 (CAP1) reduces migration in esophageal squamous cell carcinoma. Jpn J Clin Oncol 43: 856-864, 2013.

21. Liu Y, Wang Y, Cheng C, Chen Y, Shi S, Qin J, Xiao F, Zhou D, $\mathrm{Lu} \mathrm{M}, \mathrm{Lu}$ Q and Shen A: A relationship between p27(kip1) and Skp2 after adult brain injury: Implications for glial proliferation. J Neurotrauma 27: 361-371, 2010.

22. Dong C, Yuan T, Wu Y, Wang Y, Fan TW, Miriyala S, Lin Y, Yao J, Shi J, Kang T, et al: Loss of FBP1 by Snail-mediated repression provides metabolic advantages in basal-like breast cancer. Cancer Cell 23: 316-331, 2013.

23. Malz M, Weber A, Singer S, Riehmer V, Bissinger M, Riener MO, Longerich T, Soll C, Vogel A, Angel P, et al: Overexpression of far upstream element binding proteins: A mechanism regulating proliferation and migration in liver cancer cells. Hepatology 50: 1130-1139, 2009.

24. Chen M, Zhang J, Li N, Qian Z, Zhu M, Li Q, Zheng J, Wang X and Shi G: Promoter hypermethylation mediated downregulation of FBP1 in human hepatocellular carcinoma and colon cancer. PLoS One 6: e25564, 2011.

25. Singer S, Malz M, Herpel E, Warth A, Bissinger M, Keith M, Muley T, Meister M, Hoffmann H, Penzel R, et al: Coordinated expression of stathmin family members by far upstream sequence element-binding protein-1 increases motility in non-small cell lung cancer. Cancer Res 69: 2234-2243, 2009. 
26. Ding Z, Liu X, Liu Y, Zhang J, Huang X, Yang X, Yao L, Cui G and Wang D: Expression of far upstream element (FUSE) binding protein 1 in human glioma is correlated with c-Myc and cell proliferation. Mol Carcinog 54: 405-415, 2015.

27. Burger JA, Ghia P, Rosenwald A and Caligaris-Cappio F: The microenvironment in mature B-cell malignancies: A target for new treatment strategies. Blood 114: 3367-3375, 2009.

28. Fei M, Hang Q, Hou S, He S and Ruan C: Adhesion to fibronectin induces $\mathrm{p} 27$ (kip1) nuclear accumulation through down-regulation of Jab1 and contributes to cell adhesion-mediated drug resistance (CAM-DR) in RPMI 8,226 cells. Mol Cell Biochem 386: 177-187, 2014.

29. Fei M, Hang Q, Hou S and Ruan C: Cell adhesion to fibronectin down-regulates the expression of Spy1 and contributes to drug resistance in multiple myeloma cells. Int J Hematol 98: 446-455, 2013.

30. Yagi K, Yamamoto K, Umeda S, Abe S, Suzuki S, Onishi I, Kirimura S, Fukayama M, Arai A, Kitagawa M and Kurata M: Expression of multidrug resistance 1 gene in B-cell lymphomas: Association with follicular dendritic cells. Histopathology 62: 414-420, 2013

31. Kurtova AV, Tamayo AT, Ford RJ and Burger JA: Mantle cell lymphoma cells express high levels of CXCR4, CXCR5, and VLA-4 (CD49d): Importance for interactions with the stromal microenvironment and specific targeting. Blood 113: 4604-4613, 2009.
32. Guan C, Shi H, Wang H, Zhang J, Ni W, Chen B, Hou S, Yang X, Shen A and Ni R: CtBP2 contributes to malignant development of human esophageal squamous cell carcinoma by regulation of p16INK4A. J Cell Biochem 114: 1343-1354, 2013.

33. Tjin EP, Groen RW, Vogelzang I, Derksen PW, Klok MD, Meijer HP, van Eeden S, Pals ST and Spaargaren M: Functional analysis of HGF/MET signaling and aberrant HGF-activator expression in diffuse large B-cell lymphoma. Blood 107: 760-768, 2006.

34. Fernandez-Vidal A, Ysebaert L, Didier C, Betous R, De Toni F, Prade-Houdellier N, Demur C, Contour-Galcéra MO, Prévost GP, Ducommun B, et al: Cell adhesion regulates CDC25A expression and proliferation in acute myeloid leukemia. Cancer Res 66: 7128-7135, 2006

35. Wang Y, Huang Y, Xu X, Tang J, Huang X, Zhu J, Liu J, Miao X, $\mathrm{Wu}$ Y, Yang F, et al: Expression of small glutamine-rich TPR-containing protein A (SGTA) in Non-Hodgkin's Lymphomas promotes tumor proliferation and reverses cell adhesion-mediated drug resistance (CAM-DR). Leuk Res 38: 955-963, 2014.

36. He S, Huang Y, Wang Y, Tang J, Song Y, Yu X, Ma J, Wang S, Yin H, Li Q, et al: Histamine-releasing factor/translationally controlled tumor protein plays a role in induced cell adhesion, apoptosis resistance and chemoresistance in non-Hodgkin lymphomas. Leuk Lymphoma 56: 2153-2161, 2015. 